\title{
ANÁLISE DAS DIRETRIZES ESTRATÉGICAS USADAS PARA INCORPORAR A RESILIÊNCIA NOS PROCESSOS ORGANIZACIONAIS
}

\author{
Eliane Duarte Ferreira ${ }^{1}$ \\ Universidade Federal de Santa Catarina \\ eliane_dfer@hotmail.com \\ Fabiano Pamato Nunes ${ }^{2}$ \\ Universidade Federal de Santa Catarina \\ pamatonunes@gmail.com \\ Neri dos Santos ${ }^{3}$ \\ Universidade Federal de Santa Catarina \\ nerisantos@gmail.com
}

\begin{abstract}
Resumo
A capacidade das organizações para lidar com incertezas ambientais, ameaças, crises e acontecimentos inesperados depende da sua capacidade de resiliência. Este estudo analisa as diretrizes estratégicas usadas para incorporar resiliência em processos organizacionais. Trata-se de uma revisão sistemática da literatura, nas bases de dados Scopus, Web of Science e Scielo, nos últimos 5 anos com os descritores "Resilience" AND "knowledge management"; "organizational resilience" AND "knowledge management"; "organizational resilience" AND "organizational processes" foi realizada uma análise qualitativa dos 10 artigos considerados mais relevantes na pesquisa. Como resposta percebeu-se que as diretrizes variam conforme a expertise da organização e o seu propósito comercial, porém elementos como "fortalecer foco organizacional em estratégias de resposta", "equilíbrio entre planejar e improvisar processos", "estratégias da gestão do conhecimento em práticas de gestão de risco", "capacidade de antecipação", "adaptabilidade", "troca de informação", "compartilhamento do conhecimento", "eficiência", "eficácia" e "aprendizagem organizacional" são algumas diretrizes que precisam ser consideradas.
\end{abstract}

Palavras-chave: Resiliência organizacional. Processos organizacionais. Gestão do Conhecimento.

\section{ANALYSIS OF THE STRATEGIC GUIDELINES USED TO INCORPORATE RESILIENCE IN ORGANIZATIONAL PROCESSES}

\begin{abstract}
The ability of organizations to deal with environmental uncertainties, threats, crises and unexpected events depends on their resilience. This study analyzes the strategic guidelines used to incorporate resilience in organizational processes. This is a systematic review of the literature, in the databases Scopus, Web of Science and Scielo, in the last 5 years with the descriptors "Resilience" AND "knowledge management"; "organizational resilience" AND "knowledge management"; "organizational resilience" AND "organizational processes" a qualitative analysis of the 10 articles considered most relevant in the research was carried out. As an answer, it was noticed that the guidelines vary according to the organization's expertise and its business purpose, but elements such as "strengthening organizational focus on response strategies", "balance between planning and improvising processes", "knowledge management strategies in practices risk management ", anticipation capacity "," adaptability ", " information exchange ", knowledge sharing ”," efficiency ”, effectiveness ”and" organizational learning "are some guidelines that need to be considered.
\end{abstract}

Keywords: Organizational resilience. Organizational processes. Knowledge management.

\footnotetext{
${ }^{1}$ Departamento de Engenharia do Conhecimento da Universidade Federal de Santa Catarina.

${ }^{2}$ Departamento de Engenharia do Conhecimento da Universidade Federal de Santa Catarina.

${ }^{3}$ Departamento de Engenharia do Conhecimento da Universidade Federal de Santa Catarina.

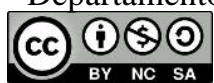

Esta obra está licenciada sob uma Licença Creative Commons (Attribution 4.0 International). P2P \& INOVAÇÃO, Rio de Janeiro, v. 6 n. 2, p.195-216, Mar./Ago. 2020. 


\section{INTRODUÇÃO}

No artigo "A primer in resilience: Maximizing value beyond earnings", publicado pela empresa McKinsey \& Company, Kevin Carmody et al (2019) expõem que empresas resilientes não podem prever o futuro, mas certamente podem preparar-se para ele.

Blake et al. (2019) apontam a resiliência organizacional como a capacidade que as empresas adquirem de resistir, absorver e adaptar-se a interrupções e retornar à funcionalidade normal. A resiliência requer improvisação, flexibilidade, adaptação e inovação às mudanças.

Os processos organizacionais que permitem a resiliência dependem fortemente da capacidade de resposta e aprendizado, por meio de processos inter-organizacionais e de detecção, antecipação, adaptação e aprendizado entre sistemas, (SUTCLIFFE;VOGUS, 2003) descrevem isso como saber o que fazer, o que procurar, o que esperar e o que aconteceu.

Os eventos que levam as organizações a resistirem às crises podem surgir de diferentes formas, por crises financeiras globais, estagnação econômica, desastres naturais, erros humanos e terrorismo (INCE et al., 2017). Independentemente da forma como surgem, é vital para as organizações tenham tenacidade e se mantenham atentas a esses eventos.

A gestão do conhecimento organizacional tem como fundamentos estruturais as pessoas, os processos e as tecnologias, por intermédio dos quais as organizações adquirem, organizam, processam informações, gerando conhecimentos, que são compartilhados, com o objetivo de gerar novos conhecimentos (SANTOS, 2005). Esse fluxo de geração de vantagem competitiva ocorre com o acionamento das capacidades organizacionais para implementação de suas estratégias (NONAKA; TAKEUCHI, 2004).

Baseado nos fundamentos teóricos da gestão do conhecimento, do gerenciamento de processos e da resiliência organizacional, este artigo tem como objetivo geral analisar as diretrizes estratégicas usadas pelas organizações para incorporar a resiliência em seus processos organizacionais. Mais especificamente, o artigo objetiva, também: (i) identificar as principais áreas temáticas; (ii) descrever os métodos de pesquisa utilizados e (iii) analisar os principais resultados obtidos.

Para o alcance desses objetivos, partiu-se da seguinte questão de pesquisa: Quais diretrizes estratégicas devem ser definidas para incorporar a resiliência nos processos organizacionais? 


\section{FUNDAMENTAÇÃO TEÓRICA}

Os fundamentos teóricos que nortearam a pesquisa estão relacionados aos temas "resiliência organizacional" e "gestão por processo", com o intuito apenas de conceituá-los, conforme abordados nesta pesquisa.

\subsection{Resiliência Organizacional}

Em ecossistemas organizacionais voláteis (BHAMRA et al., 2011) as vantagens competitivas são temporárias e as organizações enfrentam desafios para encarar dinamicamente as mudanças externas e as turbulências. Assim sendo, resiliência é a capacidade de renovar continuamente, reconstruir e sustentar-se depois de um evento incomum ou destrutivo (HOLLING, 1973).

O conceito de resiliência é abordado interdisciplinarmente em áreas que tratam de sistemas ecológicos (MCDANIELS et al., 2008), gestão de crises (SOMERS, 2009), engenharia (MADNI; JACKSON, 2009), gestão de emergências (STEPHENSON et al., 2010), gestão da cadeia de abastecimento (PONOMAROV; HOLCOMB, 2009) e gestão pública (BOIN; VAN EETEN, 2013).

No campo organizacional as pesquisas ainda são recentes, porém vem despertando interesse dos pesquisadores de forma ascendente.

Pode ser percebida como a capacidade de conceber e implementar comportamentos adaptativos positivos adequados para lidar com as tensões causadas por situações de mudança rápida (MALLAK, 1998), manter-se de forma mais eficaz do que os concorrentes em condições desfavoráveis, monitorar constantemente as oportunidades no ambiente, esforçar-se para estar pronto em determinadas situações, para obter vantagem competitiva (VOGUS; SUTCLIFFE, 2007).

Para Storesund et al. (2018) o conceito evoluiu de resiliência ecológica, via psicologia, para a engenharia no campo de redução de risco de desastres.

Norris et al. (2008) defendem que o termo se originou da física e denota a capacidade de um sistema retornar rapidamente ao equilíbrio após uma perturbação externa.

De fato, o conceito de resiliência tem origem na física, que caracteriza um material que, simultaneamente, apresenta três propriedades que, aparentemente, são incompatíveis: resistência, tenacidade e flexibilidade (SANTOS, 2019). 
No âmbito das organizações tem sido bastante comum utilizar o termo resiliência organizacional para definir capacidades sociais e culturais que contribuem para as organizações sobreviverem e, até mesmo, prosperarem em momentos de crise.

Num contexto generalista, Ince et al. (2017) afirmam que a resiliência organizacional surge quando as empresas são obrigadas a manter suas habilidades, competências e capacidades organizacionais em um ambiente em mudança, desafiando situações de competição.

Para ilustrar melhor o conceito de resiliência dos diversos autores acerca do tema algumas definições são apresentadas no quadro 01 .

Quadro 01 - Definições de Resiliência Organizacional

\begin{tabular}{|l|l|}
\hline \multicolumn{1}{|c|}{ Autores } & \multicolumn{1}{c|}{ Definições } \\
\hline Starr; Newfrock; Delurey (2003) & $\begin{array}{l}\text { Capacidade de suportar descontinuidades } \\
\text { sistêmicas e se adaptar às novas condições } \\
\text { ambientais de risco. }\end{array}$ \\
\hline Lengnick-hall; Beck (2005) & $\begin{array}{l}\text { Capacidade da organização para enfrentar e } \\
\text { superar grandes desafios. }\end{array}$ \\
\hline Boin; Comfort; Demchak (2010) & $\begin{array}{l}\text { Capacidade de "salto para trás" em caso de uma } \\
\text { perturbação. }\end{array}$ \\
\hline Mafabi; munene; ntayi (2012) & $\begin{array}{l}\text { Capacidade de uma organização para ser proativo } \\
\text { e / ou reativo para lidar com as demandas } \\
\text { ambientais e ameaças }\end{array}$ \\
\hline Ince et al. (2017) & $\begin{array}{l}\text { Capacidade das organizações para lidar com } \\
\text { incertezas ambientais, ameaças, crises e } \\
\text { acontecimentos inesperados }\end{array}$ \\
\hline Wieland; Marcus Wallenburg (2013) & $\begin{array}{l}\text { Capacidade de investir na rotina de compartilhar } \\
\text { conhecimento sobre mudanças relevantes no } \\
\text { ambiente, em avançar ou quando ocorrerem. }\end{array}$ \\
\hline Manab; aziz (2019) & $\begin{array}{l}\text { Capacidade de uma organização antecipar e } \\
\text { planejar com antecedência para lidar com eventos } \\
\text { potenciais inesperados por meio da abordagem } \\
\text { SRM para a sobrevivência da empresa a longo } \\
\text { prazo. }\end{array}$ \\
\hline
\end{tabular}

Fonte: Dados da pesquisa (2019)

Mesmo não existindo um consenso acerca das definições do termo resiliência organizacional, em virtude da interdisciplinaridade do tema, é possível constatar que alguns elementos são recorrentes em todos os construtos, como "gerir mudanças ameaças e desafios"; "adaptação ao ambiente"; "responder eficazmente a eventos ameaçadores e destrutivos" e "sobrevivência do negócio". 


\subsection{Gestão por Processos}

A literatura é repleta de definições sobre Gestão por Processos (GP). Para este trabalho considera-se que a GP é um conjunto de atividades estrategicamente pensadas a fim de criar melhoria nos processos de forma contínua e padronizada (SANTOS, 2019). A GP pode ser considerada uma evolução da Reengenharia, quando esta foi reconhecida como inapropriada já que ignorava aspectos como gestão estratégica e de pessoas, e se beneficia, também, dos métodos e ferramentas que foram utilizados até então (MELÃO, 2010).

Sampaio (2017) argumenta que GP é um conjunto de ações metodologicamente coerentes com o objetivo de melhoria contínua das organizações. Para dar suporte a esta constante melhoria é necessário que as organizações estejam abertas a inovação com o intuito de reconhecer e adequar as mudanças necessárias com a finalidade de conseguir dar maior valor ao consumidor uma vez que consegue impactar de forma mais precisa na qualidade dos seus produtos ou serviços (BRANCO et al., 2019)

Gonçalves (2000) destaca que os processos estão divididos em três categorias básicas: os processos de negócios, onde o foco é o cliente e é suportado por outros processos internos; os processos organizacionais, que garantem o sucesso da coordenação de subprocessos interligados que garantem o funcionamento da organização e consequentemente os processos de negócios; e os processos gerenciais que contribuem para o relacionamento entre os gerentes e também para mediação e ajustes de desempenho.

De Vrieze e Xu (2018) relatam que os sistemas de processos, quando interligados, além de garantirem os processos de negócio da organização também trazem propriedades de resiliência dos processos. Uma vez que haja independência do controle dos processos adjacentes, uma falha total no sistema se torna mais difícil em caso de falhas nos sistemas que o compõem. Ainda, segundo os autores, esta resiliência nos processos precisa ser pensada na criação do sistema.

\section{PROCEDIMENTO METODOLÓGICO}

O procedimento metodológico de pesquisa utilizado para a realização desta pesquisa foi a revisão integrativa.

Souza et al. (2018), definem esse procedimento como um estudo com coleta de dados realizada a partir de fontes secundárias, por meio de levantamento bibliográfico e baseado na experiência vivenciada pelos autores, por ocasião da realização de uma revisão integrativa. 
Assim, esta pesquisa se divide em seis fases, sendo que a estrutura do artigo está composto, conforme proposto por Souza et al., (2018) a saber:

$\underline{\mathbf{1}^{a} \text { fase: }}$ elaboração da pergunta norteadora;

$\underline{2^{\mathbf{a}} \text { fase: }}$ busca ou amostragem na literatura;

$\underline{\mathbf{3}^{\mathbf{a}} \text { fase: }}$ coleta de dados;

$\underline{4^{\mathbf{a}} \text { fase: }}$ análise crítica dos estudos incluídos;

$\underline{5^{\mathbf{a}} \text { fase: }}$ discussão dos resultados;

$\underline{\mathbf{6}^{\mathbf{a}} \text { fase: }}$ apresentação da revisão integrativa.

Nesta perspectiva, foi formulada a seguinte pergunta norteadora de pesquisa: Quais diretrizes estratégicas devem ser definidas para incorporar a resiliência nos processos organizacionais?

Assim sendo, a pesquisa realizada teve como objetivo geral analisar as diretrizes estratégicas usadas para incorporar a resiliência nos processos organizacionais em estudos empíricos, na literatura nacional e internacional. Mais especificamente, a pesquisa teve os seguintes objetivos: (i) identificar as principais áreas temáticas; (ii) descrever os métodos de pesquisa utilizados e (iii) analisar os objetivos e os principais resultados obtidos.

A busca sistemática na literatura e a coleta de dados, deu-se no dia 12 de outubro de 2019 e os passos efetuados seguem descritos no Quadro 02, abaixo:

Quadro 02 - Descrição dos passos realizados na busca sistemática

\begin{tabular}{|c|c|}
\hline Estratégia & Descrição \\
\hline Bases de dados & $\begin{array}{l}\text { - Scopus - campo: article, title, abstract, keyword } \\
\text { - Web foi Science - campo: Tópico } \\
\text { - Scielo (português, espanhol) - campo: todos os índices }\end{array}$ \\
\hline Descritores & $\begin{array}{l}\text { - Resilience AND "knowledge management"; "organizational resilience" } \\
\text { AND "knowledge management"; "organizational resilience" } \\
\text { AND "organizational processes" }\end{array}$ \\
\hline Tipo de documento & $\begin{array}{l}\text { - Artigos de periódico indexados } \\
\text { - Artigos publicados em anais de evento indexados } \\
\text { - Teses e dissertações }\end{array}$ \\
\hline Língua & - Inglês, espanhol e português \\
\hline $\begin{array}{l}\text { Critérios de } \\
\text { inclusão }\end{array}$ & $\begin{array}{l}\text { - Estudos empíricos } \\
\text { - Acesso ao documento completo } \\
\text { - Foco em organizações }\end{array}$ \\
\hline \begin{tabular}{ll|} 
Critério & de \\
Exclusão &
\end{tabular} & $\begin{array}{l}\text { - Documentos duplicados } \\
\text { - Publicações anteriores a } 2015\end{array}$ \\
\hline
\end{tabular}

Fonte: Dados da pesquisa (2019) 
A análise crítica e o processo de seleção dos estudos incluídos foram desenvolvidos em duas etapas: (i) leitura prévia dos títulos e resumos, aplicando-se os filtros de inclusão. Essa etapa gerou o grupo de artigos pré-selecionados e (ii) leitura completa das produções préselecionadas, aplicando-se os filtros de exclusão.

Para a discussão dos resultados foi realizada um processo de análise do conteúdo das publicações selecionadas. Para tanto, organizou-se uma matriz de síntese com os conteúdos a serem analisados conforme os objetivos propostos. Os resultados alcançados seguem descritos no item 4 (Indicadores Bibliométricos). Neste item, apresentam-se sequencialmente análises contextuais bibliométricas dos artigos, seguido de análises qualitativas e das considerações finais.

A apresentação dos resultados da pesquisa realizada para seleção dos artigos é apresentada na Tabela 01 .

Tabela 01 - Síntese dos resultados da pesquisa para seleção dos artigos

\begin{tabular}{l|c|c|c} 
Base & Recuperados & Pré-selecionados & Selecionados \\
Scopus & 200 & & 8 \\
\hline Web of Science & 69 & 24 & 4 \\
\hline Scielo & 1 & & 3 \\
\hline Duplicados & & 55 & 10 \\
\hline Total & 270 & & \\
\hline \multicolumn{2}{r|}{ Fonte: Dados da pesquisa (2019) }
\end{tabular}

O processo de seleção dos artigos foi desenvolvido em duas etapas: na primeira etapa foi realizada a leitura prévia dos títulos e resumos, aplicando-se os filtros de inclusão. Essa etapa gerou o grupo de pré-selecionados e, na segunda etapa, foi realizada a leitura completa dos artigos e aplicou-se os filtros de exclusão, conforme forme demonstrados no Tabela 02, abaixo. 
Tabela 02 - Publicações selecionadas para análise

\begin{tabular}{|c|c|c|c|c|}
\hline ID & Ano & País & Autoria & Título \\
\hline 1 & 2019 & Japão & $\begin{array}{l}\text { Azadegan, A } \\
\text { Srinivasan, R } \\
\text { Blome, C }\end{array}$ & $\begin{array}{l}\text { Learning from near-miss events: An organizational } \\
\text { learning perspective on supply chain disruption } \\
\text { response }\end{array}$ \\
\hline 2 & 2019 & Noruega & $\begin{array}{l}\text { Bento, F } \\
\text { Garotti, L }\end{array}$ & $\begin{array}{l}\text { Resilience beyond formal structures: A network } \\
\text { perspective towards the challenges of an aging } \\
\text { workforce in the oil and gas industry }\end{array}$ \\
\hline 3 & 2019 & $\begin{array}{c}\text { Nova } \\
\text { Zelândia }\end{array}$ & $\begin{array}{c}\text { Blake, D M } \\
\text { Stevenson, J } \\
\text { Wotherspoon, L }\end{array}$ & $\begin{array}{l}\text { The role of data and information exchanges in } \\
\text { transport system disaster recovery: A New Zealand } \\
\text { case study }\end{array}$ \\
\hline 4 & 2019 & Bangladesch & $\begin{array}{c}\text { Jilani, Munshi Muhammad } \\
\text { Abdul Kader } \\
\text { Fan, Luo } \\
\text { Nusrat, Mansura } \\
\text { Uddin, Md. Aftab }\end{array}$ & $\begin{array}{l}\text { Empirical Study on the Antecedents Predicting } \\
\text { Organizational Resilience of Small and Medium } \\
\text { Enterprises in Bangladesh }\end{array}$ \\
\hline 5 & 2019 & Uganda & $\begin{array}{l}\text { Manab, N A } \\
\text { Aziz, N A A }\end{array}$ & $\begin{array}{l}\text { Integrating knowledge management in } \\
\text { sustainability risk management practices for } \\
\text { company survival }\end{array}$ \\
\hline 6 & 2019 & Turquia & $\begin{array}{l}\text { Şengül, H } \\
\text { Marşan, D } \\
\text { Gün, T } \\
\end{array}$ & $\begin{array}{l}\text { Survey assessment of organizational resiliency } \\
\text { potential of a group of Seveso organizations in } \\
\text { Turkey }\end{array}$ \\
\hline 7 & 2018 & EUA & $\begin{array}{c}\text { Burnard, Kevin } \\
\text { Bhamra, Ran } \\
\text { Tsinopoulos, Christos }\end{array}$ & $\begin{array}{l}\text { Building Organizational Resilience: Four } \\
\text { Configurations }\end{array}$ \\
\hline 8 & 2018 & Brasil & $\begin{array}{c}\text { Sincorá, Larissa Alves } \\
\text { Oliveira, Marcos Paulo } \\
\text { Valadares de } \\
\text { Zanquetto-Filho, Hélio } \\
\text { Ladeira, Marcelo Bronzo }\end{array}$ & $\begin{array}{l}\text { Business analytics leveraging resilience in } \\
\text { organizational processes }\end{array}$ \\
\hline 9 & 2017 & Japão & $\begin{array}{c}\text { Shoji, } \mathrm{N} \\
\text { Iwaasa, T } \\
\text { Nakajima, Y } \\
\text { Mizuno, M }\end{array}$ & $\begin{array}{l}\text { The way to use the guidelines for supporting } \\
\text { resilience enhancement: From verifying } \\
\text { effectiveness using before-after comparison test } \\
\text { design of two fitness centers }\end{array}$ \\
\hline 10 & 2017 & Dinamarca & Dalgaard-Nielsen, A. & $\begin{array}{l}\text { Organizational resilience in national security } \\
\text { bureaucracies: Realistic and practicable? }\end{array}$ \\
\hline
\end{tabular}

Fonte: Dados da pesquisa (2019)

\section{INDICADORES BIBLIOMÉTRICOS}

Visando facilitar a contextualização em relação aos estudos selecionados optou-se por retratar alguns dados bibliométricos. Para iniciar apresentam-se os dados referentes à autoria, periódicos e datas de publicação dos artigos. Em seguida mostram-se um quadro referente à localização geográfica das produções e um mapeamento dos principais temas abordados nos trabalhos.

\subsection{Quanto a Autoria, Periódicos a Datas de Publicação}

Quanto a autoria, periódicos e datas de publicação, pode-se evidenciar os seguintes aspectos: 
Em relação a autoria, quase todas as publicações possuem autoria múltipla, apenas um único artigo (Organizational resilience in national security bureaucracies: Realistic and practicable?) é de autoria individual, os demais artigos foram elaborados por 2 autores ou mais. Observou-se que o continente asiático se destaca em primeiro lugar em relação ao número de pesquisadores. Foram encontrados 10 autores a saber: Azadegan, A; Srinivasan, R; Blome, C; Jilani, Munshi Muhammad Abdul Kader; Fan, Luo; Nusrat, Mansura; Uddin, Md. Aftab; Shoji, N Iwaasa, T; Nakajima, Y; Mizuno, M; Şengül, H; Marşan, D Gün, T. Em segundo lugar aparecem os continentes Europeu e Americano com 7 pesquisadores cada e a Oceania com 2 pesquisadores.

Em relação aos periódicos, observou-se que os artigos foram publicados em revistas das áreas de ciências Sociais e interdisciplinares: International Journal of Production Economics; Proceedings of the Institution of Mechanical Engineers, Journal of Risk and Reliability; IEEE Transactions on Engineering Management; Advances in Intelligent Systems and Computing; International Journal of Production Economics; Management Science Letters; RAUSP Management Journal; International Journal of Disaster Risk Reduction; Journal of Open Innovation: Technology, Market, and Complexity; 2016 International Annual Conference of the American Society for Engineering Management, ASEM 2016; Lecture Notes in Business Information Pro.

Em relação aos anos de publicação, enquadrados nos limites desta pesquisa, em que se buscou publicações a partir de 2015, evidenciou-se que, nos anos de 2017 e 2018, houve 4 publicações e, em 2018, foram publicados 3 artigos e, finalmente, em 2019, até a data desta pesquisa, verificou-se 6 publicações. Assim, observa-se que, em 2017 e 2018, as publicações se mantiveram num ritmo e, em 2019, tem sido mais ascendente, percebendo-se que existe uma tendência crescente de publicações nesta área.

\subsection{Quanto às Áreas Temáticas}

Quanto as áreas temáticas, as pesquisas realizadas na área de resiliência organizacional abrangem diferentes contextos e, neste artigo, elas foram agrupadas da seguinte forma:

Estratégias de resposta organizacional sobre interrupção na cadeia de suprimentos (AZADEGAN et al., 2019; BLAKE et al., 2019; BURNARD; BHAMRA; TSINOPOULOS, 2018); Interação com a Gestão do conhecimento - (BENTO; GAROTTI, 2019; JILANI et al., 2019a; MANAB; AZIZ, 2019; SINCORÁ et al., 2018); dilemas gerenciais entre equilibrar resiliência, austeridade - (DALGAARD-NIELSEN, 2017); Aprendizagem organizacional - 
(ŞENGÜL; MARŞAN; GÜN, 2019); Eficácia nas diretrizes para aplicar resiliência - (SHOJI et al., 2017).

\subsection{Quanto aos Métodos De Pesquisa}

Quanto aos métodos de pesquisa utilizados, procurou-se estruturar um quadro, contendo os diferentes autores, abordagem e técnica de pesquisa utilizadas, assim como o público alvo e a amostra de pesquisa, conforme o Quadro 03.

Em relação à abordagem, procurou-se evidenciar se ela foi quantitativa ou qualitativa.

Em relação à técnica de pesquisa, para coleta de dados, procurou-se evidenciar se ela foi realizada por questionário ou por entrevista.

Em relação ao público alvo, procurou-se evidenciar qual era o foco, se o nível estratégico, gerencial ou operacional da organização.

Em relação à amostra, procurou-se evidenciar o seu tamanho e representatividade. 
Quadro 03 - Métodos de pesquisa utilizados

\begin{tabular}{|c|c|c|c|}
\hline Autoria & Abordagem & Técnica & Público-alvo e amostra \\
\hline $\begin{array}{c}\text { Azadegan, A; Srinivasan, } \mathrm{R} \\
\text { Blome, C }\end{array}$ & Quantitativa & Questionários & $\begin{array}{c}\text { Executivos seniores e gerentes } 1000 \\
\text { empresas, empresas de manufatura da } \\
\text { Suécia, Suíça e Alemanha } 448 \\
\text { questionários com retorno de } 14,0 \%\end{array}$ \\
\hline Bento, F; Garotti, L & Qualitativa & Entrevista & $\begin{array}{l}\text { Engenheiros de uma indústria de petróleo e } \\
\text { gás brasileira }\end{array}$ \\
\hline $\begin{array}{l}\text { Blake, D M; Stevenson, J; } \\
\text { Wotherspoon, L }\end{array}$ & Qualitativa & Entrevista & $\begin{array}{l}57 \text { atores de } 35 \text { organizações de transportes } \\
\text { (rodoviário, ferroviário, aéreo e marítimo) }\end{array}$ \\
\hline $\begin{array}{c}\text { Jilani, Munshi Muhammad } \\
\text { Abdul Kader; } \\
\text { Fan, Luo } \\
\text { Nusrat, Mansura } \\
\text { Uddin, Md. Aftab }\end{array}$ & Quantitativa & Questionário & $\begin{array}{l}\text { Survey com } 480 \text { questionários, utilizando } \\
\text { escala Likert de } 5 \text { pontos com variação da } \\
\text { da escala desde concordo plenamente (1) } \\
\text { para discordo fortemente (5). Foram } \\
\text { recebidas } 343 \text { respostas. Destas, } 13 \text { foram } \\
\text { descartadas por erros ou resposta nulas, } \\
\text { perfazendo um total de } 330 \text { respostas } \\
\text { válidas. Análise dos dados através dos } \\
\text { softwares Microsoft Excel 2016, AMOS } \\
\text { versão-20, e versão } 21 \text {-ferramentas IBM } \\
\text { SPSS analíticos. }\end{array}$ \\
\hline $\begin{array}{l}\text { Manab, N A } \\
\text { Aziz, N A A }\end{array}$ & Quantitativa & Questionário & $\begin{array}{l}\text { Empresas da Malásia listados que são } \\
\text { ambientalmente sensíveis e serviços. } \\
200 \text { questionários distribuídos entre os } \\
\text { participantes, dos quais } 88 \text { questionários } \\
\text { foram devolvidos, conseguindo-se assim } \\
\text { uma taxa de resposta de } 44 \text { por cento. }\end{array}$ \\
\hline $\begin{array}{l}\text { Şengül, H } \\
\text { Marşan, D } \\
\text { Gün, T }\end{array}$ & Quantitativa & Questionário & $\begin{array}{l}\text { E-mail para as pessoas de contato das } \\
\text { organizações no banco de dados Bekra que } \\
\text { consistia de } 548 \text { contatos: Um total de } 39 \\
\text { respostas eram obtido, com a maioria das } \\
\text { organizações do sector químico e petróleo } \\
\text { (32 organizações). As organizações sete } \\
\text { restantes eram de vidro e cerâmica (1), } \\
\text { defesa (1), produtos florestais (2), cimento } \\
\text { (1) e (2) os setores têxtis. }\end{array}$ \\
\hline $\begin{array}{c}\text { Burnard, Kevin } \\
\text { Bhamra, Ran } \\
\text { Tsinopoulos, Christos }\end{array}$ & Qualitativa & Entrevista & $\begin{array}{l}\text { Entrevistas, observações e documentos } \\
\text { organizacionais de três organizações do } \\
\text { Reino Unido no sector da energia. }\end{array}$ \\
\hline $\begin{array}{c}\text { Sincorá, Larissa Alves } \\
\text { Oliveira, Marcos Paulo } \\
\text { Valadares de } \\
\text { Zanquetto-Filho, Hélio } \\
\text { Ladeira, Marcelo Bronzo }\end{array}$ & Quantitativa & Questionário & $\begin{array}{l}\text { Gestores das empresas vinculadas a } \\
\text { FINDES, com } 49 \text { questões (escala Likert) } \\
\text { com } 89 \text { respostas válidas }\end{array}$ \\
\hline $\begin{array}{c}\text { Shoji, } \mathrm{N} \\
\text { Iwaasa, T } \\
\text { Nakajima, Y } \\
\text { Mizuno, M }\end{array}$ & Quantitativa & Questionário & 49 Colaboradores de 2 academias do Japão \\
\hline Dalgaard-Nielsen, A. & Qualitativa & Entrevista & $\begin{array}{l}\text { Doze executivos-chefes da burocracia } \\
\text { nacional de segurança da Dinamarca. }\end{array}$ \\
\hline
\end{tabular}

Fonte: Dados da pesquisa (2019)

\section{ANÁLISE DOS RESULTADOS}

A análise dos resultados das diferentes publicações coletadas no levantamento bibliográfico realizado, nos permite responder à questão de pesquisa formulada, relativa à 
definição das diretrizes estratégicas que devem ser definidas para incorporar a resiliência nos processos organizacionais.

Nesse sentido, foi realizada uma análise de conteúdo de cada uma das publicações coletadas, procurando-se evidenciar os objetivos da pesquisa, a fundamentação teórica utilizada, a abordagem metodológica, público-alvo e os principais resultados em termos de diretrizes estratégicas sugeridas pelos autores para incorporar a resiliência nos processos organizacionais.

Assim sendo, Azadegan et al. (2019) apresentaram como objetivos de sua pesquisa, investigar se a exposição quase acidentes (near miss), que se reflete em estratégias de resposta organizacional para fornecer interrupções na cadeia suprimentos. Os autores aplicam a teoria do aprendizado organizacional relacionada ao aprendizado em loop duplo (ARGYRIS, 1976) para explicar como resultam quase erros em estratégias de resposta flexíveis ou processuais. Ao fazer isso, analisaram os efeitos gerais e sutis de considerar quase-acidentes em gerenciar interrupções na cadeia de suprimentos e, assim, determinar sua eficácia competitividade como estratégia de mitigação na melhoria da resiliência da cadeia de suprimentos. Para tanto utilizaram o método de pesquisa qualitativa, tendo como público alvo executivos seniores e gerentes envolvidos no fornecimento parcerias em cadeia em empresas de manufatura da Suécia, Suíça e Alemanha. Foram selecionadas aleatoriamente 1000 empresas em cada país. A coleta de dados aconteceu através do envio de 448 questionários, e gerou um retorno de $14,9 \%$ de respostas. Os resultados indicam que a exposição a eventos near miss levam as empresas a fortalecerem seu foco em estratégias de resposta processual e diminuir seu foco em estratégias de resposta flexíveis. Este estudo estende o corpo do gerenciamento de interrupção da cadeia de suprimentos ao conceito de quase acidentes e explica como o contexto institucional desempenha um papel importante na aprendizagem da cadeia de suprimentos respostas de interrupção. Quanto as diretrizes estratégicas sugeridas pelos autores para incorporar a resiliência nos processos organizacionais, observou-se "fortalecer foco organizacional em estratégias de resposta processual" e "diminuir seu foco em estratégias de resposta flexíveis".

$\mathrm{Na}$ mesma perspectiva, na seara da preocupação com a interrupção na cadeia de suprimentos, Blake et al. (2019) apresentam como objetivo de sua pesquisa, caracterizar como o sistema de transporte, a infraestrutura e a cadeia de suprimentos, respondem e se adaptam às interrupções relacionadas a terremotos. Para tanto realizaram entrevistas com 57 atores de 35 organizações de transportes (rodoviário, ferroviário, aéreo e marítimo). Como resultados foi verificado que trocas de informações foram eficazes, permitindo que o sistema de transporte respondesse e se adaptasse com sucesso, além de permitir a mobilidade contínua de usuários e 
mercadorias. As organizações que respondem às interrupções de transporte se basearam nas fontes de dados existentes de novas maneiras, coletaram novos conjuntos de dados e alavancaram relacionamentos para gerenciar trocas de informações. Quando verificado quais diretrizes estratégicas são mencionadas para incorporar a resiliência nos processos organizacionais, percebeu-se que "compartilhamento de experiências", "troca de informações e dados", "colaboração", "comunicação eficaz" são fortemente destacadas neste estudo.

Os estudos de Bento e Garotti (2019) buscaram analisar a crise da perda de conhecimento por motivos de aposentadoria ou da rotatividade na perspectiva da resiliência da rede em sistemas complexos. Como procedimentos metodológicos, realizaram uma pesquisa qualitativa, com engenheiros de uma indústria de petróleo e gás brasileira. Como resultado da pesquisa, eles evidenciaram que o conhecimento estruturado da rede pode ajudar as empresas de petróleo e gás a enfrentar os desafios do envelhecimento da força de trabalho de pelo menos duas maneiras: primeiro, antecipando possíveis riscos de perda de conhecimento, compreendendo os papéis aposentados em sua estrutura de rede, que fornece informações valiosas sobre o estado inicial do sistema, antecipando possíveis cenários e, segundo, promovendo a estrutura de rede resiliência, entendendo os processos locais de adaptação, mas também pontes por intermédio das quais informações fluam sob a forma de ideias, normas e cooperação social. Como diretrizes estratégicas para incorporar a resiliência nos processos organizacionais pode-se perceber "adaptabilidade", “memória organizacional", “conhecimentos explícitos e tácitos integrados".

Os estudos de Burnard et al. (2018) propõem explorar empiricamente os processos organizacionais desde o início das interrupções e explorar os fatores que determinam diferentes configurações de construção de resiliência. Os dados foram coletados por meio de entrevistas, observações e documentos organizacionais de três organizações do Reino Unido no sector da energia. Identificar a configuração da resiliência é parte do posicionamento estratégico de uma organização em relação às exigências de seu ambiente operacional. No entanto, a tomada de decisão durante uma resposta é realizada, principalmente, no nível operacional. Como resultado, os gerentes procuram equilibrar o desejo de implementar um plano de gestão de crises com a vontade de improvisar e alocar recursos rapidamente. Embora as organizações possam adotar uma abordagem estratégica, as exigências e restrições impostas, após a retomada de uma interrupção, significa o que eles devem considerar para gerenciar cada fase durante a escalada ou o desenvolvimento de uma interrupção. Como diretrizes estratégicas para incorporar a resiliência nos processos organizacionais, os autores mencionam “equilibrar o desejo de 
implementar um plano de gestão de crises" com a "vontade de improvisar e alocar recursos rapidamente".

Dalgaard-Nielsen (2017) apresentou como objetivo de sua pesquisa explorar os dilemas gerenciais que procuram equilibrar resiliência, austeridade fiscal e responsabilidade democrática. $\mathrm{O}$ artigo mostra como os executivos procuram introduzir elementos de resiliência organizacional por meio de "reservas virtuais" e planos de contingência gerais e flexíveis. O caso dinamarquês indicou a necessidade de uma reavaliação crítica das condições de contorno da resiliência organizacional quando se trata de organizações do setor público. A presença generalizada de resiliência como objetivo e solução nos discursos de segurança nacional pode servir para inspirar ajustes organizacionais relevantes, como redes mais externas, diversidade de recursos internos e colaboração em meio de equipes. As diretrizes estratégicas para incorporar a resiliência nos processos organizacionais, percebidas neste estudo são "eficiência", "controle hierárquico com o exercício da autoridade pública e consistência" e "previsibilidade na administração de regras".

Manab e Aziz (2019) apresentam uma contribuição teórica e prática para a compreensão do efeito moderador da gestão do conhecimento sobre a sobrevivência da empresa. Os resultados apontam que a resiliência organizacional é crucial para ajudar as empresas a se preparar para eventos negativos em um ambiente de negócios incerto. A descoberta afirma que a gestão do conhecimento age como um recurso estratégico da empresa para apoiar uma melhor identificação e mitigação de riscos de eventos potenciais futuros que, em seguida, garantir a sobrevivência da empresa. Como diretrizes estratégicas para incorporar a resiliência nos processos organizacionais, a serem seguidas, sugerem "estratégica da gestão do conhecimento em práticas de gestão de risco e sustentabilidade", para mitigar os impactos inesperados de riscos emergentes.

Sengül et al. (2019), em um estudo realizado no Grupo SEVESO da Turquia, procuraram avaliar o potencial de resiliência organizacional, por meio de uma pesquisa, utilizando a escala Likert de 5 pontos, projetados para medir quatro dimensões importantes de capacidades dinâmicas, incluindo capacidades de antecipação, cultura adaptativa, recursos de rede e aprendizagem organizacional. Os resultados do estudo revelaram que existem diferenças significativas no potencial de resiliência organizacional entre as organizações, nessas quatro dimensões. Apesar das organizações participantes terem marcado potenciais relativamente altos de resiliência, as áreas que mais necessitam de melhorias, são aumento antecipação e recursos de rede que fortalecem o envolvimento dos funcionários para aumentar ainda mais a resiliência das organizações. Dessa forma os autores deixam como diretrizes estratégicas para incorporar 
a resiliência nos processos organizacionais: "capacidade de antecipação", "cultura adaptativa", "recursos de rede" e "aprendizagem organizacional".

Jilani et al. (2019), em um estudo empírico, realizado em empresas de Bangladesh, avaliaram se as variáveis "clima criativo", "resiliência dos funcionários" e a "gestão do conhecimento" afetam organizações resilientes. Os resultados apontam que essas variáveis possuem efeito nas organizações resilientes em diferentes níveis. A "resiliência dos empregados" e o "clima criativo", assim como a "gestão do conhecimento", são importantes diretrizes estratégicas para incorporar a resiliência nos processos organizacionais. Entretanto, diferentemente do que encontraram na literatura, a gestão do conhecimento não afeta significativamente a resiliência dos funcionários. Em contrapartida, o clima criativo tem um efeito insignificante em organizações resilientes em Bangladesh. Em vista disso, eles acreditam que o tamanho da amostra tenha sido um fator limitante do estudo e pode ter desvirtuado este resultado.

Sincorá et al. (2018), em um estudo realizado no estado do Espírito Santo (Brasil), procurando responder a seguinte questão de pesquisa: "a capacidade analítica organizacional pode influenciar o relacionamento entre a maturidade de gerenciamento de processos de uma empresa e a sua resiliência organizacional?’. Assim, essas relações são estudadas utilizando uma amostra de micro, pequenas, médias, médias e grandes empresas do estado do Espírito Santo, atuando em diferentes segmentos da indústria, comércio e serviços. Os resultados confirmaram as hipóteses teóricas dos autores ao atestar que estimular a prática de análise de dados e informações de rotina organizacional levam a um comportamento gerencial relevante. Isso se dá porque o "desenvolvimento do conhecimento" e o "entendimento de como gerenciar eventos de riscos inesperados" pode levar a empresa a reagir de forma mais precisa, mesmo em falhas operacionais.

Shoji et al. (2016), em um estudo realizado, definiram como objetivo de seu artigo verificar a eficácia de diretrizes estratégicas para o apoio a melhoria da resiliência e discutir qual a melhor maneira de usar essas diretrizes baseando-se em dados estatísticos. Os resultados apontam que fatores como "otimismo", "controle", "vitalidade", "tentativas de resolver problemas", "auto entendimento" e "empatia" podem ser considerados como diretrizes estratégicas para incorporar a resiliência nos processos organizacionais. Após o primeiro teste, foi promovido um encontro com os gerentes para passar como abordar o aprimoramento da resiliência. Em um segundo encontro foi explicado como utilizar as diretrizes e confirmado o seu uso. A partir desses encontros, os gerentes e os funcionários realizaram as atividades para aprimorar a resiliência. Cada uma das academias teve um tempo diferente para aplicar as 
diretrizes. A Escala Bidimensional de Resiliência (BRS) foi utilizada para avaliar a resiliência, com questionário utilizando a escala Likert com 5 níveis. Para a análise dos resultados foi utilizado o software de estatística SPSS v-21. Houve uma diferenciação entre os resultados da academia A e da academia B, onde uma delas utilizou as diretrizes estratégicas para uma melhoria da performance como um todo, enquanto na outra houve uma busca por desenvolvimento de habilidades individuais. Estes resultados encontrados pelos pesquisadores levaram a uma proposta de uma nova categorização de avaliação. Esta nova categorização pode ser usada e desenvolvida como uma estrutura em pesquisas futuras. A pesquisa revelou que o potencial de resiliência das organizações respondentes é bastante alto. Entretanto os pesquisadores temem que os resultados possam ser tendenciosos, uma vez que as organizações pesquisadas já são bem estabelecidas.

Após a análise dos artigos selecionados e identificadas as principais diretrizes estratégicas utilizadas para incorporar a resiliência nos processos organizacionais, os principais resultados obtidos por esta pesquisa estão demonstrados na figura 01, abaixo:

Figura 1- estratégias para incorporar resiliência nos processos organizacionais

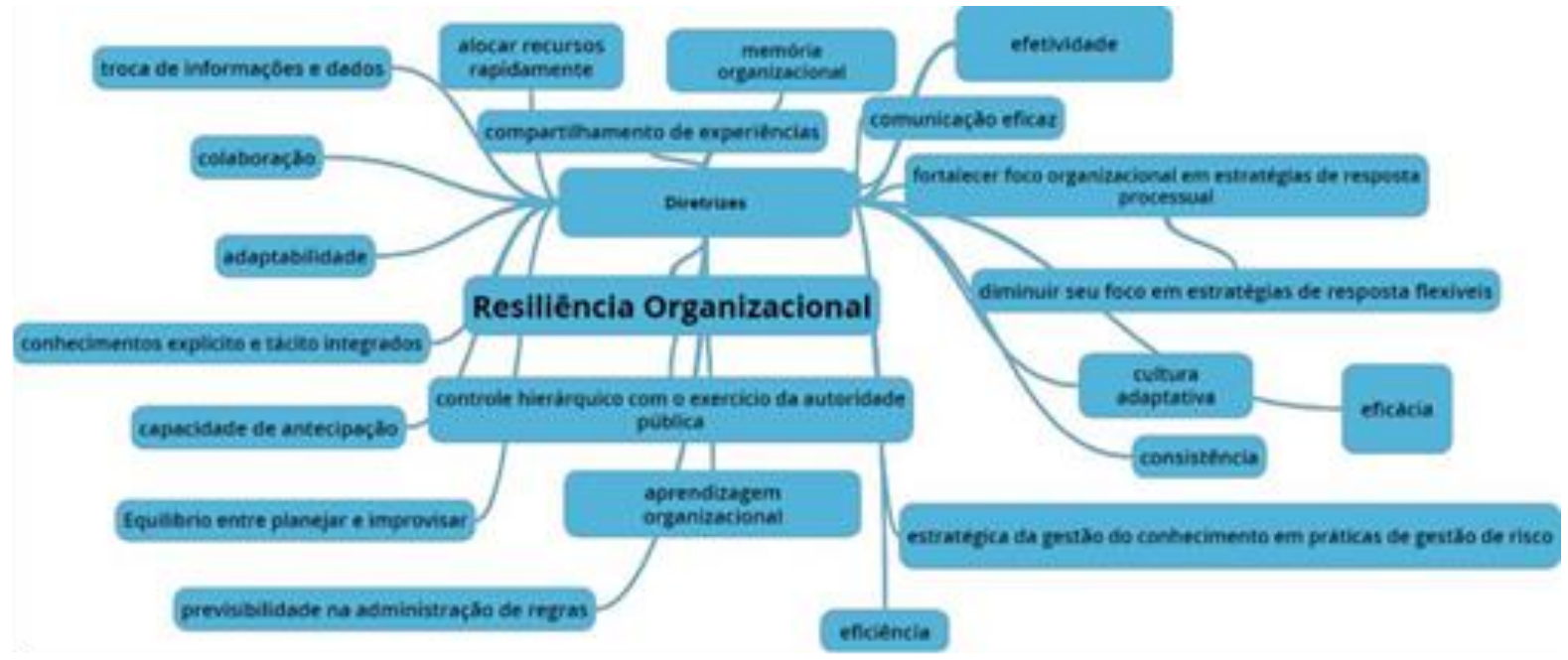

Fonte: Dados da pesquisa. Ferramenta web utilizada: GoConqr. Disponível em: 〈https://www.goconqr.com>. Acesso em: 29 nov. de 2019.

Conforme mostra a figura 01, a síntese dos resultados sugere que a resiliência pode ser incorporada nos processos organizacionais, conforme as considerações evidenciadas nos artigos analisados:

A organização de todo mundo tem encarado a resiliência como um processo organizacional e não apenas como um resultado após a recuperação, e estão voltadas a responder continuamente aos desafios e fornecer serviços valiosos e ininterruptos à sociedade; 
Que apesar de todas as organizações apresentam interesse pelo tema resiliência, por estarem preocupadas com a sobrevivência no mercado, grande maioria, ainda foca em eventos relativos a desastres e pós desastres e como sobreviver a esses estes, mitigando a interrupção na cadeia de suprimentos;

Que as diretrizes sugeridas pelos autores para incorporar a resiliência nos processos organizacionais variam conforme a expertise da organização e o seu proposito comercial, mas que independente desses pressupostos todos estão interessadas em se preparar para o futuro, pois compreendem que impossível prevê-lo;

Que os principais achados no que se refere as diretrizes estratégicas sugeridas pelos autores para incorporar a resiliência nos processos organizacionais são: "fortalecer foco organizacional em estratégias de resposta processual e diminuir seu foco em estratégias de resposta flexíveis"; "compartilhamento de experiências", "troca de informações e dados", "colaboração", “comunicação eficaz”, “adaptabilidade", "memória organizacional”, “conhecimentos explícitos e tácitos integrados"; "equilíbrio entre o desejo de implementar um plano de gestão de crises com a vontade de improvisar e alocar recursos rapidamente"; "eficiência", "controle hierárquico com o exercício da autoridade pública"; "consistência e previsibilidade na administração de regras"; "estratégica da gestão do conhecimento em práticas de gestão de risco e sustentabilidade para mitigar os impactos inesperados de riscos emergentes"; "capacidade de antecipação"; "cultura adaptativa"; "recursos de rede" e "aprendizagem organizacional".

\section{CONSIDERAÇÕES FINAIS}

O quadro que se apresenta neste trabalho é revelador. Apesar de ser um fenômeno relativamente recente, os estudos empíricos de incorporação da resiliência organizacional nos processos organizacionais estão crescendo ao redor do mundo. Pode-se notar que pouco tem sido produzido academicamente no Brasil, mas há um destaque para a produção acadêmica na Ásia.

No que se refere ao termo "resiliência", ele tem sido definido em diversas áreas do conhecimento, como na física, nas ciências dos materiais e na engenharia. Contudo, na medida que este conceito passe a ser incorporado pelas ciências organizacionais, novas definições são elaboradas, dando uma contribuição importante para a sobrevivência das organizações em tempos de incerteza e de elevada competitividade. À medida que os conceitos e os resultados de estudos sobre a incorporação da resiliência em processos organizacionais são obtidos, novos 
trabalhos científicos serão necessários para acompanhar o andamento da implementação desses resultados e, assim sendo, contribuir para melhoria do mundo dos negócios.

No que se refere aos achados relativos à pergunta de pesquisa, formulada nesta pesquisa, que buscava evidenciar "quais diretrizes estratégicas deveriam ser formuladas para incorporar resiliência nos processos organizacionais?" constata-se que a resposta à esta pergunta, varia conforme a expertise da organização e o seu propósito comercial, porém várias pistas, tais como: "fortalecer foco organizacional em estratégias de resposta", "equilíbrio entre planejar e improvisar processos", "estratégias de gestão do conhecimento em práticas de gestão de risco", "capacidade de antecipação", "adaptabilidade", "troca de informação", "compartilhamento do conhecimento", "eficiência organizacional", "eficácia organizacional" e "aprendizagem organizacional" podem ser delineadas como algumas diretrizes estratégicas que precisam ser consideradas para incorporar resiliência nos processos organizacionais.

Acredita-se que este artigo poderá contribuir para que futuros pesquisadores possam encontrar uma aplicação dos procedimentos metodológicos que foram utilizados e das diretrizes estratégicas que foram evidenciadas, em outras pesquisas empíricas e, assim sendo, dar sustentabilidade conceitual a seus trabalhos futuros, podendo comparar a teoria com práticas já aplicadas nos negócios. 


\section{REFERENCIAS}

AZADEGAN, A. et al. Learning from near-miss events: an organizational learning perspective on supply chain disruption response. International Journal of Production Economics, v. 216, p. 215-226, 2019.

BENTO, F.; GAROTTI, L. Resilience beyond formal structures: a network perspective towards the challenges of an aging workforce in the oil and gas industry. Journal of Open Innovation: Technology, Market, and Complexity, v. 5, n. 1, 2019.

BHAMRA, R.; DANI, S.; BURNARD, K. Resilience: the concept, a literature review and future directions. International Journal of Production Research, v. 49, n. 18, p. 53755393, 2011.

BLAKE, D. M. et al. The role of data and information exchanges in transport system disaster recovery: a New Zealand case study. International Journal of Disaster Risk Reduction, v. $39,2019$.

BOIN, A.; COMFORT, L. K.; DEMCHAK, C. C. Designing resilience: preparing for extreme events. University of Pittsburgh Press, 2010.p. 1-12.

BOIN, A.; VAN EETEN, M. J. G. The resilient organization. Public Management Review, v.15, n. 3, p. 429-445, 2013.

BRANCO, A. N. et al. Gerenciamento de processos de negócio e lean thinking como base para a melhoria na gestão do patrimônio em uma instituição pública de Ensino Superior.

Revista Gestão da Produção, Operações e Sistemas, v. 14, n. 2, p. 75-94, 2019.

BURNARD, K.; BHAMRA, R.; TSINOPOULOS, C. Building Organizational Resilience: four configurations. IEEE Transactions on Engineering Management, v. 65, n. 3, p. 351362, ago. 2018.

DALGAARD-NIELSEN, A. Organizational resilience in national security bureaucracies:

Realistic and practicable? Journal of Contingencies and Crisis Management, v. 25, n. 4, p. 341-349, dez. 2017.

DE VRIEZE, P.; XU, L. Resilience analysis of service-oriented collaboration process management systems. Service Oriented Computing and Applications, v. 12, n. 1, p. 25-39, 2018.

GONÇALVES, J. E. L. As Empresas são grandes coleções de processos. Revista de Administração de Empresas, v. 40, n. 1, p. 6-19, 2000.

HOLLING, C. S. Resilience and stability of ecological systems. Annual review of ecology and systematics, v. 4, n. 1, p. 1-23, 1973.

INCE, H. et al. The Search for Understanding Organizational Resilience. In:13 The international strategic management, 2017. Anais...Trukey, 2017. 
JILANI, M. M. A. K. et al. Empirical Study on the Antecedents Predicting Organizational Resilience of Small and Medium Enterprises in Bangladesh. Revista de Inovação e Sustentabilidade, v. 10, n. 2, p. 138-145, 2019a.

JILANI, M. M. A. K. et al. Empirical Study on the Antecedents Predicting Organizational Resilience of Small And Medium Enterprises In Bangladesh. Revista de Inovação e Sustentabilidade, v. 10, n. 2, p. 138-145, 2019 b.

LENGNICK-HALL, C. A.; BECK, T. E. Adaptive fit versus robust transformation: How organizations respond to environmental change. Journal of Management, v. 31, n. 5, p. 738 757, 2005.

MADNI, A. M.; JACKSON, S. Towards a conceptual framework for resilience engineering. IEEE Systems Journal, v. 3, n. 2, p. 181-191, 2009.

MAFABI, S.; MUNENE, J.; NTAYI, J. Knowledge management and organisational resilience: Organisational innovation as a mediator in Uganda parastatals. Journal of Strategy and Management, v. 5, n. 1, p. 57-80, 2012.

MALLAK, L. Putting organizational resilience to work. Industrial Management-Chicago then Atlanta-, p. 8-13, 1998.

MANAB, N. A.; AZIZ, N. A. A. Integrating knowledge management in sustainability risk management practices for company survival. Management Science Letters, v. 9, n. 4, p. 585- 594, 2019.

MCDANIELS, T. et al. Fostering resilience to extreme events within infrastructure systems: Characterizing decision contexts for mitigation and adaptation. Global Environmental Change, v. 18, n. 2, p. 310-318, 2008.

MELÃO, N.; PIDD, M. A conceptual framework for understanding business processes and business process modelling. Info Systems Journal, v. 10, n. 2, p. 105-129, 2010.

NONAKA, I.; TAKEUCHI, H. Criação de conhecimento na empresa. Rio de Janeiro: Elsevier, 2004.

NORRIS, F. H. et al. Community resilience as a metaphor, theory, set of capacities, and strategy for disaster readiness. American journal of community psychology, v. 41, n. 1-2, p. $127-150,2008$.

PONOMAROV, S. Y.; HOLCOMB, M. C. Understanding the concept of supply chain resilience. The international journal of logistics management, v. 20, n. 1, p. 124-143, 2009.

SAMPAIO, T. L. Mapeamento do conhecimento nos processos de rotina de laboratório de microbiologia clínica. 91 f. Dissertação (Mestrado) - Curso de Engenharia e Gestão do Conhecimento, Universidade Federal de Santa Catarina, Florianópolis, 2017.

SANTOS, J. S. C. Colaboração na gestão de processos de negócios: proposta de um framework para implantação do social BPM. 169 f. Dissertação (Mestrado)- Curso de Administração,

Universidade Federal de Sergipe, São Cristóvão, 2019. 
SANTOS, N., Gestão estratégica do conhecimento. Apostila do Programa de PósGraduação em Engenharia e Gestão do Conhecimento, UFSC, Florianópolis, 2005.

SANTOS, N. Transformação Digital: Da Gestão do Conhecimento à Inovação Contínua. In: IX Congresso Internacional de Conhecimento e Inovação, 2019. Anais...Porto Alegre: UFSC, 2019.

ŞENGÜL, H.; MARŞAN, D.; GÜN, T. Survey assessment of organizational resiliency potential of a group of Seveso organizations in Turkey. Proceedings of the Institution of Mechanical Engineers, Part O: journal of risk and reliability, v. 233, n. 3, p. 470-486, 2019.

SHOJI, N. et al. The way to use the guidelines for supporting resilience enhancement: from verifying effectiveness using before-after comparison test design of two fitness centers.

Advances in social \& occupational ergonomics, v. 487, p. 435-445, 2016.

SHOJI, N. et al. The way to use the guidelines for supporting resilience enhancement: from verifying effectiveness using before-after comparison test design of two fitness centers. Advances in intelligent systems and computing, v. 487, p. 435-445, 2017.

SINCORÁ, L. A. et al. Business analytics leveraging resilience in organizational processes. RAUSP Management Journal, v. 53, n. 3, p. 385-403, 9 jul. 2018.

SOMERS, S. Measuring resilience potential: an adaptive strategy for organizational crisis planning. Journal of contingencies and crisis management, v. 17, n. 1, p. 12-23, 2009.

SOUZA, Marcela Tavares de; SILVA, Michelly Dias da and CARVALHO, Rachel de. Integrative review: what is it? How do it? Einstein (São Paulo), vol.8, n. 1, pp.102-106. mar.2010. FapUNIFESP (SciELO). http://dx.doi.org/10.1590/s1679-45082010rw1134

STARR, R.; NEWFROCK, J.; DELUREY, M. Enterprise resilience: managing risk in the networked economy. Strategy and Business, v. 30, p. 70-79, 2003.

STEPHENSON, A.; VARGO, J.; SEVILLE, E. Measuring and comparing organisational resilience in Auckland. Australian journal of emergency management, v. 25, n. 2, p. 27 32, 2010.

STORESUND, K. et al. Novel methodologies for analysing critical infrastructure resilience. In: 28th International European Safety and Reliability Conference, 2018. Anais... Norway: CRC Press/Balkema, 2018.

SUTCLIFFE, K. M.; VOGUS, T. J. Organizing for resilience. Positive organizational scholarship: foundations of a new discipline, v. 94, p. 110, 2003.

VOGUS, T. J.; SUTCLIFFE, K. M. Organizational resilience: towards a theory and research agenda. In: International Conference on Systems, Man and Cybernetics, 2007. Anais...IEEE, 2007.

WIELAND, A.; MARCUS WALLENBURG, C. The influence of relational competencies on supply chain resilience: a relational view. International journal of physical distribution \& logistics management, v. 43, n. 4, p. 300-320, 2013. 
- INOVAÇão

\section{REFERÊNCIA UTILIZADA}

JACOBS, KEVIN CARMODY, CLIFFORD CHEN, S. A primer in resilience : maximizing value beyond earnings. nov, 2019. 